\title{
Effect of Perpendicular Magnetic Field on Free Convection in a Rectangular Cavity
}

\author{
${ }^{\#}$ Anand Kumar ${ }^{1}$, Ashok K. Singh ${ }^{1}$, Pallath Chandran ${ }^{2 \star}$ and Nirmal C. Sacheti ${ }^{2}$
}

\begin{abstract}
${ }^{1}$ Department of Mathematics, Faculty of Science, Banaras Hindu University, Varanasi-221005, India. "Present Address: Department of Mathematics, Central University of Rajasthan, Ajmer, Rajasthan, India. ${ }^{2}$ Department of Mathematics and Statistics, College of Science, Sultan Qaboos University, P.O. BOX: 36, PC 123, Al Khod, Muscat, Sultanate of Oman. *Email: chandran@squ.edu.om.
\end{abstract}

\begin{abstract}
The steady free convective flow of a viscous incompressible and electrically conducting fluid in a two-dimensional cavity in the presence of a magnetic field applied normal to the plane of the cavity is investigated. The side vertical walls of the cavity are heated differentially while the horizontal walls are assumed to be insulated. The governing equations are re-formulated in terms of vorticity and stream function. The resulting boundary value problem is solved numerically using an alternating direction implicit (ADI) method. A number of plots illustrating the influence of Hartmann number and Rayleigh number on the streamlines and isotherms as well as the velocity and temperature profiles are shown. Furthermore, results for the average Nusselt number and the maximum absolute stream function have been obtained, and these are compared with the corresponding results in the literature when the magnetic field is applied along the cavity in the horizontal direction.
\end{abstract}

Keywords: Cavity flow; Free convection; Magnetic field; Stream function; Vorticity.
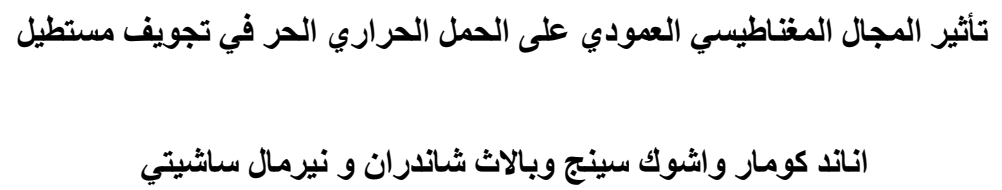

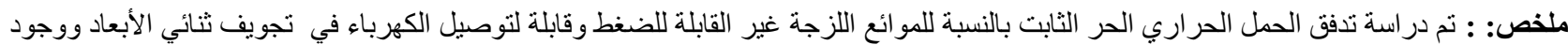

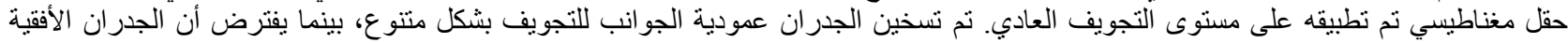

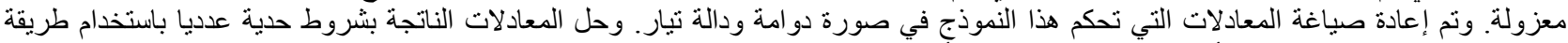

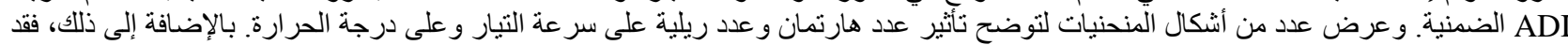

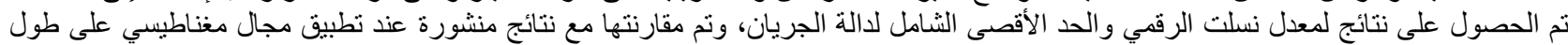
تجويف في الاتجاه الأفقي.

$$
\text { كلمات مفتاحية: الحمل الحراري الحر، مجال مغناطيسي، تدفق في جوف، دالة التيار، الدوامة . }
$$

\section{Introduction}

$\mathbf{T}$

The theoretical and experimental studies of laminar free convective flows in channels and enclosures have a number of applications, for instance in the cooling of reactors and electronic equipment, crystal growth in liquids, storage systems and solar technology, etc. Batchelor [1] initiated investigations on natural convection in an enclosure heated and cooled from side walls. He concluded that the flow is determined by three key dimensionless parameters, namely, the Prandtl number, the Rayleigh number and the aspect ratio of the cavity. Following Batchelor's work, a number of researchers carried out experimental and numerical investigations related to natural convection in vertical enclosures. Eckert and Carlson [2] and Elder [3] experimentally studied natural convection in a rectangular cavity across which a temperature differential occurred. Elder [4] subsequently obtained numerical solutions of steady free convective flow in a vertical slot and compared the numerical results with the experimental findings [2,3]. Gill [5] presented approximate solutions of equations governing natural convection in a rectangular cavity for the case of large values of Prandtl number. He found his results in satisfactory agreement with the experimental results of Elder [3]. Wilkes and Churchill [6] and later de Vahl Davis [7] introduced a numerical computation model to discuss free convection in a rectangular cavity. Kimura and Bejan [8] discussed the free convective flow in a rectangular cavity assuming uniform 
heat influx from one side of the cavity. In this work, the importance of heat flux wall thermal condition was highlighted and described in detail. Numerical and theoretical investigations of the transient natural convection in a 2-dimensional rectangular enclosure were carried out by Hall et al. [9]. These authors assumed one of the side walls to be heated suddenly while keeping the remaining three walls insulated. Some other aspects of convection in electrically nonconducting fluids in vertical channels and enclosures-mixed convection, porous media flow, stability, anisotropy - were discussed by Straughan [10], Hill and Straughan [11], Barletta et al. [12], Oztop et al. [13], and Tiwari et al. [14]. A detailed exposition of stability and nonlinear convection is also available in the research monograph of Straughan [15].

In the above studies, the flow features in the cavities were reported in the absence of a magnetic field. Flows of electrically conducting fluids subject to the influence of an externally applied magnetic field have been analyzed in the literature extensively due to their applications, for instance, in the manufacturing industry. This has led to a number of researchers carrying out investigations on the hydromagnetic free convective flows taking place inside rectangular enclosures or in vertical channels, albeit with some simplifying assumptions. Oreper and Szekely [16] carried out a numerical investigation for transient flow in a rectangular cavity in the presence of an imposed magnetic field and noted that the magnetic field suppresses the natural convection. Garandet et al. [17] carried out an analytical study for hydromagnetic free convection in a rectangular cavity. Rudraiah et al. [18] considered the effect of a transverse magnetic field while assuming the vertical walls of the rectangular cavity to be isothermal and horizontal walls to be insulated. A similar study to that in [18] was reported by Alchaar et al. [19]. Kanafer and Chamkha [20] investigated the effect of heat generation on hydromagnetic natural convection from an inclined porous square enclosure. Pirmohammadi et al. [21] have dealt with the effect of an applied magnetic field on a buoyancy-driven flow in a differentially heated square cavity. These authors have obtained solutions of the flow problem for wide-ranging values of Rayleigh and Hartmann numbers assuming the applied magnetic field to be along the plane of the flow. The transient nature of such a flow has heen discussed by these authors in a subsequent work [22]. In order to analyse the effect of the inclination of a rectangular vertical cavity on the buoyancy driven flows of electrically conducting fluids in the presence of applied magnetic field, a number of researchers have carried out numerical studies [23-26]. Another aspect of magneto-free convective flow in a partitioned enclosure has been dealt with by Pirmohammadi and Ghassani [27]. They have shown that the partition of an enclosure can cause the flow to separate and then form vortices inside the cavity. In a subsequent work, Pirmohammadi et al. [28] have extended some of their previous works on hydromagnetic convection in a rectangular enclosure to include the temperature dependent variations of thermal conductivity, specific heat capacity, fluid viscosity and electrical conductivity.

In the present work, we analyze the free convective flow of an electrically conducting fluid in a rectangular vertical cavity under the influence of a magnetic field applied normal to the plane of the cavity. It is worth mentioning here that in most of the experimental or theoretical two-dimensional natural convection studies involving a magnetic field, the magnetic field has been assumed to be applied in the plane of the flow-either perpendicular to or along the direction of gravity. Thus, the flow configuration considered herein results in the magnetic coupling of the momentum equations, necessitating the consideration of both the horizontal and vertical components of the velocity. It is assumed that the magnetic Reynolds number is small. The governing hydromagnetic equations, under Boussinesq approximation, are solved numerically by using a computationally stable ADI method. The effects of the transport parameters on the streamlines, isotherms, and also the velocity and temperature are analyzed. The results of the present study are compared with those of Pirmohammadi et al. [21], and have enabled us to highlight the distinguishing effect of the direction of application of the magnetic field. The results reported here have particular relevance to applications involving control of convection.

It may be mentioned here that in [21], the authors used a control-volume based finite volume method to solve the field equations directly. However, in our case, we have used a vorticity-stream function formulation of the governing equations which, in turn, has been solved using an appropriate ADI method, as explained above.

\section{Mathematical Formulation}

Consider a steady free-convective flow of a viscous incompressible and electrically conducting fluid in a rectangular cavity of length $L$ and width $H$. The $x^{\prime}$ - axis is taken along the lower horizontal wall and $y^{\prime}-$ axis transverse to it in the plane of the cavity, having origin at the lower end of the left wall as shown in Figure 1. A constant magnetic field of magnitude $B_{0}$ is applied normal to the cavity in the $z^{\prime}$ - direction. As stated earlier, the flow is at small magnetic Reynolds number so that the induced magnetic field can be neglected. The top and bottom walls of the cavity are thermally insulated while the vertical left and right walls are maintained at constant but different temperatures $T_{h}^{\prime}$ and $T_{c}^{\prime}$, respectively. Furthermore, the viscous and Ohmic dissipation terms are assumed to be negligible. 


\section{EFFECT OF PERPENDICULAR MAGNETIC FIELD ON FREE CONVECTION}

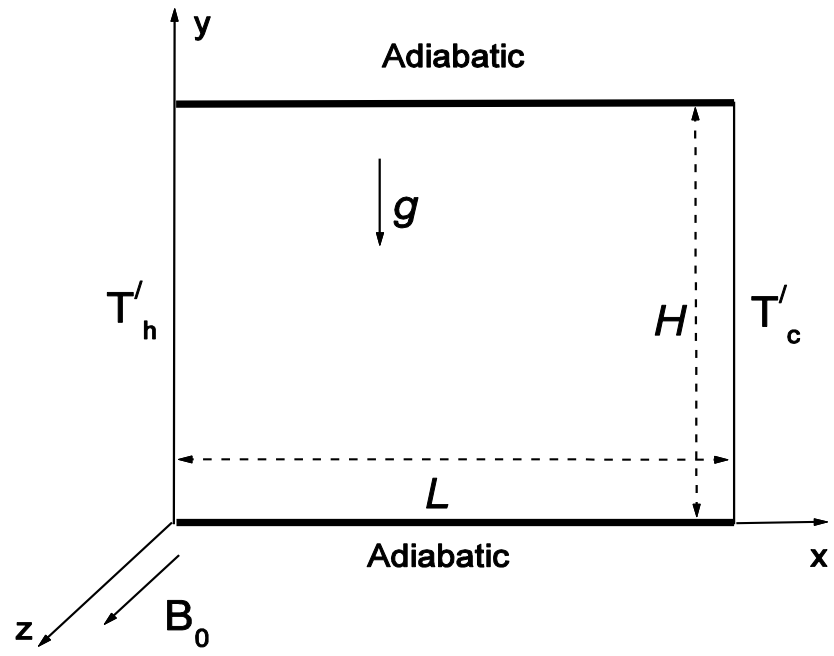

Figure 1. Physical configuration.

Under the Boussinesq approximation, the governing equations - continuity, momentum and energy - in dimensional form, can be written as

$$
\begin{aligned}
& \frac{\partial u^{\prime}}{\partial x^{\prime}}+\frac{\partial v^{\prime}}{\partial y^{\prime}}=0, \\
& u^{\prime} \frac{\partial u^{\prime}}{\partial x^{\prime}}+v^{\prime} \frac{\partial u^{\prime}}{\partial y^{\prime}}=-\frac{1}{\rho} \frac{\partial p^{\prime}}{\partial x^{\prime}}+v \nabla^{2} u^{\prime}-\frac{\sigma B_{0}^{2}}{\rho} u^{\prime}, \\
& u^{\prime} \frac{\partial v^{\prime}}{\partial x^{\prime}}+v^{\prime} \frac{\partial v^{\prime}}{\partial y^{\prime}}=-\frac{1}{\rho} \frac{\partial p^{\prime}}{\partial y^{\prime}}+v \nabla^{2} v^{\prime}+g \beta\left(T^{\prime}-T_{c}^{\prime}\right)-\frac{\sigma B_{0}^{2}}{\rho} v^{\prime}, \\
& u^{\prime} \frac{\partial T^{\prime}}{\partial x^{\prime}}+v^{\prime} \frac{\partial T^{\prime}}{\partial y^{\prime}}=\alpha \nabla^{2} T^{\prime},
\end{aligned}
$$

where $u^{\prime}$ and $v^{\prime}$ are the velocity components in the $x^{\prime}$ and $y^{\prime}$ directions, respectively, $p^{\prime}$ the pressure, $T^{\prime}$ the temperature, $v$ the kinematic viscosity, $\alpha$ the thermal diffusivity, $\sigma$ the electrical conductivity and $\beta$ is the coefficient of volumetric expansion.

For the flow model considered, the boundary conditions for the velocity and temperature fields are given by

$$
\begin{gathered}
u^{\prime}=v^{\prime}=0 \text { for } 0 \leqslant x^{\prime} \leqslant L, 0 \leqslant y^{\prime} \leqslant H, \\
T^{\prime}=T_{h}^{\prime} \text { at } x^{\prime}=0, \quad T^{\prime}=T_{c}^{\prime} \text { at } x^{\prime}=L, \\
\frac{\partial T^{\prime}}{\partial y^{\prime}}=0 \text { at } y^{\prime}=0 \text { and } y^{\prime}=H .
\end{gathered}
$$

In order to recast the momentum equations (2) and (3) in terms of vorticity $\zeta^{\prime}$ and stream function $\psi^{\prime}$, we introduce

$$
\zeta^{\prime}=\frac{\partial v^{\prime}}{\partial x^{\prime}}-\frac{\partial u^{\prime}}{\partial y^{\prime}}, \quad\left(u^{\prime}, v^{\prime}\right)=\left(\frac{\partial \psi^{\prime}}{\partial y^{\prime}},-\frac{\partial \psi^{\prime}}{\partial x^{\prime}}\right) .
$$

Introducing the nondimensional variables

$$
\begin{aligned}
& (x, y)=\left(x^{\prime}, y^{\prime}\right) / L, \quad(u, v)=\left(u^{\prime}, v^{\prime}\right) L / \alpha, \quad \zeta=L^{2} \zeta^{\prime} / \alpha, \\
& \psi=\psi^{\prime} / \alpha, \quad T=\left(T^{\prime}-T_{c}^{\prime}\right) /\left(T_{h}^{\prime}-T_{c}^{\prime}\right),
\end{aligned}
$$

the quantities in (6) take the dimensionless forms

$$
\zeta=\frac{\partial v}{\partial x}-\frac{\partial u}{\partial y}, \quad(u, v)=\left(\frac{\partial \psi}{\partial y},-\frac{\partial \psi}{\partial x}\right)
$$

while (2)-(4) can be expressed as

$$
u \frac{\partial \zeta}{\partial x}+v \frac{\partial \zeta}{\partial y}=\operatorname{Pr} \nabla^{2} \zeta+\operatorname{RaPr} \frac{\partial T}{\partial x}-\mathrm{Ha}^{2} \operatorname{Pr} \zeta
$$




$$
\begin{aligned}
& u \frac{\partial T}{\partial x}+v \frac{\partial T}{\partial y}=\nabla^{2} T, \\
& \nabla^{2} \psi=-\zeta .
\end{aligned}
$$

In (9)-(11), we have introduced a number of well-known nondimensional parameters, namely, the Rayleigh number (Ra), Prandtl number $(\mathrm{Pr})$ and Hartmann number $(\mathrm{Ha})$. These are defined as

$$
\mathrm{Ra}=g \beta\left(T_{h}^{\prime}-T_{c}^{\prime}\right) L^{3} /(v \alpha), \quad \operatorname{Pr}=v / \alpha, \mathrm{Ha}^{2}=\sigma B_{0}^{2} L^{2} /(\rho v) .
$$

In view of (7), the set of boundary conditions (5) can be shown to transform to

$$
\begin{gathered}
\psi=0 \quad \text { on } x=0,1 ; \quad y=0, y=\mathrm{Ar} \\
\zeta \simeq-\frac{\partial^{2} \psi}{\partial x^{2}} \quad \text { on } x=0 \text { and } x=1 \\
\zeta \simeq-\frac{\partial^{2} \psi}{\partial y^{2}} \quad \text { on } y=0 \text { and } y=\mathrm{Ar} \\
T=1-x \quad \text { on } x=0 \text { and } x=1 \\
\frac{\partial T}{\partial y}=0 \quad \text { on } y=0 \text { and } y=\mathrm{Ar}
\end{gathered}
$$

where the aspect ratio $\mathrm{Ar}$ is defined as $\mathrm{Ar}=H / L$.

\section{Numerical Procedure}

The transport equations (9)-(11) are highly nonlinear, and their solutions, subject to the boundary conditions (13)-(17), are obtained numerically. To facilitate this, the partial differential equations are first transformed into the parabolic form by adding false transient terms [29]. The solutions of the transformed equations are obtained by employing a uniform grid discretization and then solving the resulting finite difference equations by a well-known alternating direction implicit (ADI) method [30]. Finally, the system of linear algebraic equations, expressed tridiagonally, has been solved.

In the computational procedure, we have taken the square grid, $101 \times 101$, in order to obtain accurate results. In each iteration process, firstly the temperature field is obtained by using the values of the temperature and velocity components obtained in the previous iteration. These values are then used to compute the vorticity and the stream function, respectively. Finally, the velocity components are obtained by using the newly obtained values of the stream function. The solution procedure from the initial state is iterated until a quasi-steady state is approached by satisfying the convergence criterion

$$
\sum_{i, j}\left|\frac{\Phi_{i, j}^{n+1}-\Phi_{i, j}^{n}}{\Phi_{i, j}^{n+1}}\right|<10^{-6} .
$$

In the above expression $\Phi$ may stand for the temperature, vorticity or stream function. The superscripts denote the values of the dependent variables after the $n$th and $(n+1)$ th iterations, respectively, whereas the subscripts $i$ and $j$ indicate grid location in the $x y$-plane. In order to validate the accuracy of our computational procedure, we have compared our results in the absence of a magnetic field with the corresponding results reported by Pirmohammadi et al. [21], and found good agreement.

In engineering applications, one of the important characteristics of the flow is the rate of heat transfer across the cavity, and this is estimated by computing the values of the Nusselt number, Nu. The local Nusselt number on the left wall of the cavity is defined by

$$
\mathrm{Nu}=\left(\frac{\partial T}{\partial x}\right)_{x=0}
$$

The overall heat transfer rate across the cavity, expressed by the average Nusselt number at the left vertical wall, is

$$
\mathrm{Nu}_{\mathrm{av}}=\int_{0}^{\mathrm{A} r} \mathrm{Nu} \mathrm{d} y
$$

\section{Results and Discussion}

In this section, we present numerical results for two-dimensional hydromagnetic natural convection in an electrically conducting fluid inside a cavity caused by differentially heated vertical walls in the presence of a magnetic field applied normal to the plane of the cavity. It may be observed that the dimensionless equations which govern the behavior of the considered model contain four physical parameters, namely $\mathrm{Ra}, \mathrm{Ha}$, Pr and Ar. The presence of all four physical parameters in a model makes the computation and the subsequent analysis a tedious job. As we are 


\section{EFFECT OF PERPENDICULAR MAGNETIC FIELD ON FREE CONVECTION}

mainly interested in examining the effects of the magnetic field and buoyancy force on the convective flow, we have carried out computations for the Rayleigh number Ra ranging from $10^{4}$ to $10^{6}$ and the Hartmann number Ha from 0 to 100 , for specific values of the Prandtl number $\mathrm{Pr}=0.733$ and aspect ratio $\mathrm{Ar}=1$.
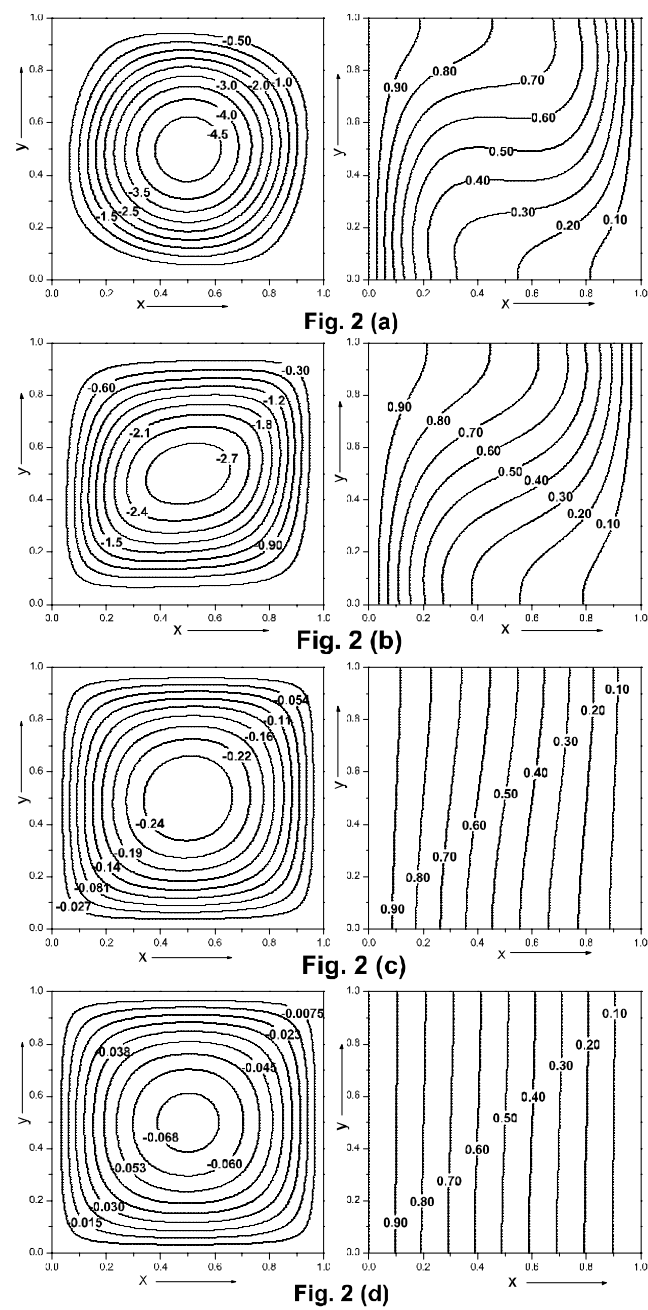

Figure 2. Streamlines and isotherms. $\mathrm{R} a=10^{4}, \mathrm{Ha}=0,10,50,100$ (a-d).

Figure 2 shows the effect of an applied magnetic field on the streamlines and isotherms as Ha increases from 0 to 100, with Ra fixed at $10^{4}$. In the absence of the applied magnetic field [Figure 2a], the flow shows a typical circulating pattern with the centre of circulation being in the middle of the cavity. The isotherms, particularly in the central portion, i.e., away from the boundaries, exhibit appreciable effects of the convection currents. However, with a moderate increase in the magnitude of the magnetic field [Figure 2b], the streamlines begin to distort from their circular shape tending to be slightly elliptic. However, the plots of streamlines and isotherms in Figure 2 seem to suggest that at this chosen level of buoyancy $\left(\mathrm{Ra}=10^{4}\right)$, isotherms are more affected in comparison to streamlines as we transit from a weak magnetic field domain to a relatively stronger magnetic field zone. In particular, at $\mathrm{Ha}=50$ or 100 , the flow is dominated by conduction, as clearly evidenced from the isotherms being almost parallel to the vertical walls. In other words, the temperature stratification, apparently visible in the case of a weak magnetic field, is no longer there, having been significantly suppressed by the applied magnetic field corresponding to higher values of Ha. This last observation is broadly similar to those reported by Rudraiah et al. [18] and Pirmohammadi et al. [21] although these studies considered the applied magnetic field to be along the cavity, parallel and transverse to the direction of gravity, respectively. However, the typical feature related to the elongation of the innermost streamline and leading to the formation of two secondary loops (see [18] and [21]), at higher values of Ha, is not observed in our work. Apparently, this difference can be attributed to the direction of the applied magnetic field being normal to the plane of the cavity in our present work as opposed to the field being along the cavity, as stated above. 

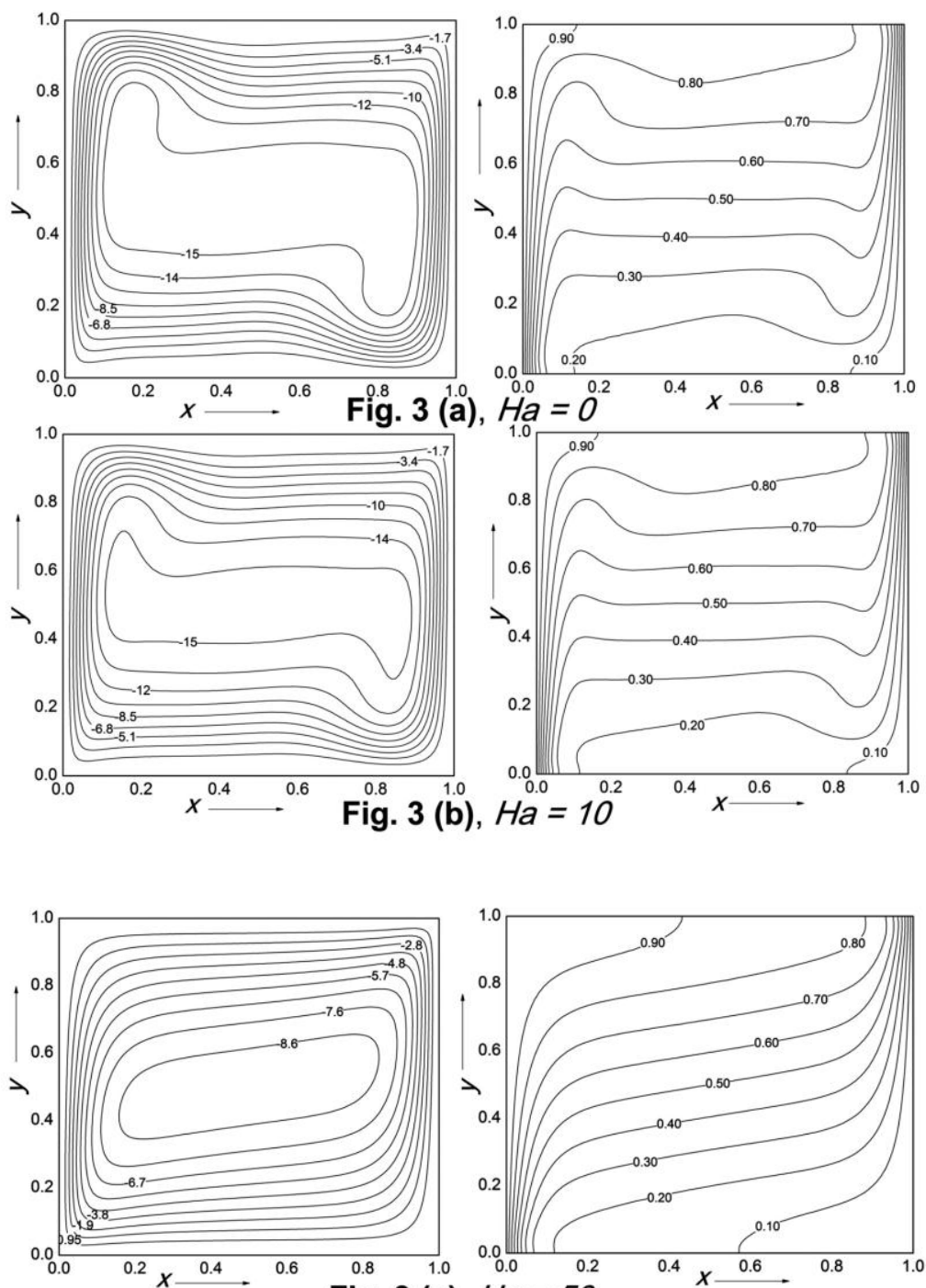

Fig. 3 (c), $\mathrm{Ha}=50$
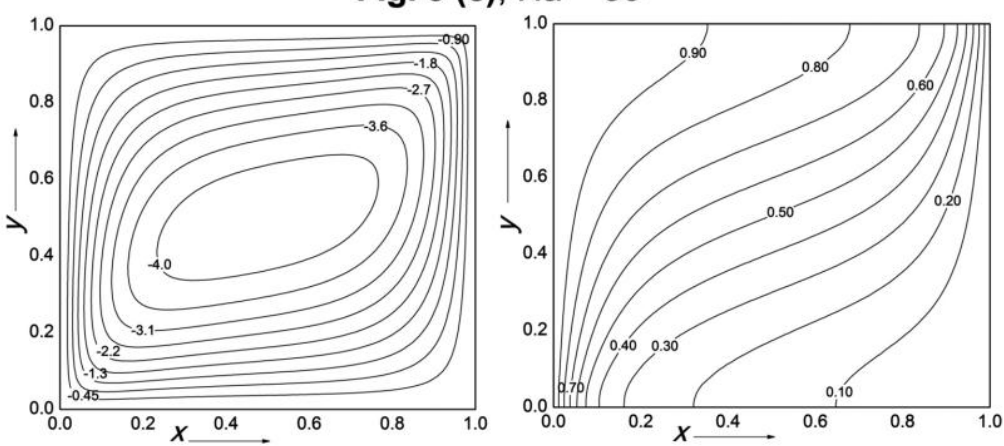

Fig. 3 (d), $\mathrm{Ha}=100$

Figure 3. Streamlines and isotherms. $\mathrm{R} a=10^{6}, \mathrm{Ha}=0,10,50,100$ (a-d).

The contours of isotherms and streamlines, for a relatively high Rayleigh number $\left(\mathrm{Ra}=10^{6}\right)$, are shown for the same set of values of Ha in Figure 3. For a weak magnetic field, the presence of thermal boundary layers along the vertical side walls is quite conspicuous. Furthermore, there is thermal stratification in the vertical direction indicating the predominance of convection currents in the heat transfer process. As regards streamlines, the circular pattern observed earlier for $\mathrm{Ra}=10^{4}$ is quite distorted [see Figure 3a or 3b]. Also, there is a strong upward or downward flow near the isothermal walls. For high values of Ha (50 or 100), the streamlines appear to gradually head towards circulating pattern through elongated ellipses (see Figure $3 \mathrm{c}$ or $3 \mathrm{~d}$ ]. Interestingly, the temperature stratification, referred to earlier for the weak magnetic field case, seems to lose its intensity in the central core region of the cavity along with the disappearance of thermal boundary layers near the side vertical walls. 


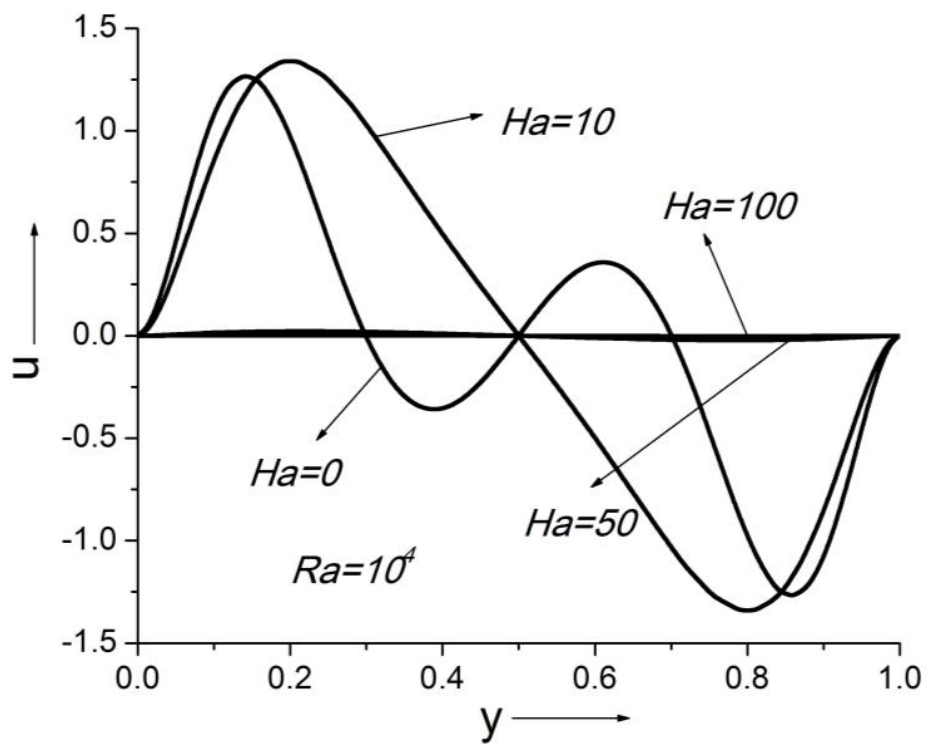

Figure 4. Velocity profiles $u$ at $x=0.5\left(\mathrm{Ra}=10^{4}\right)$.

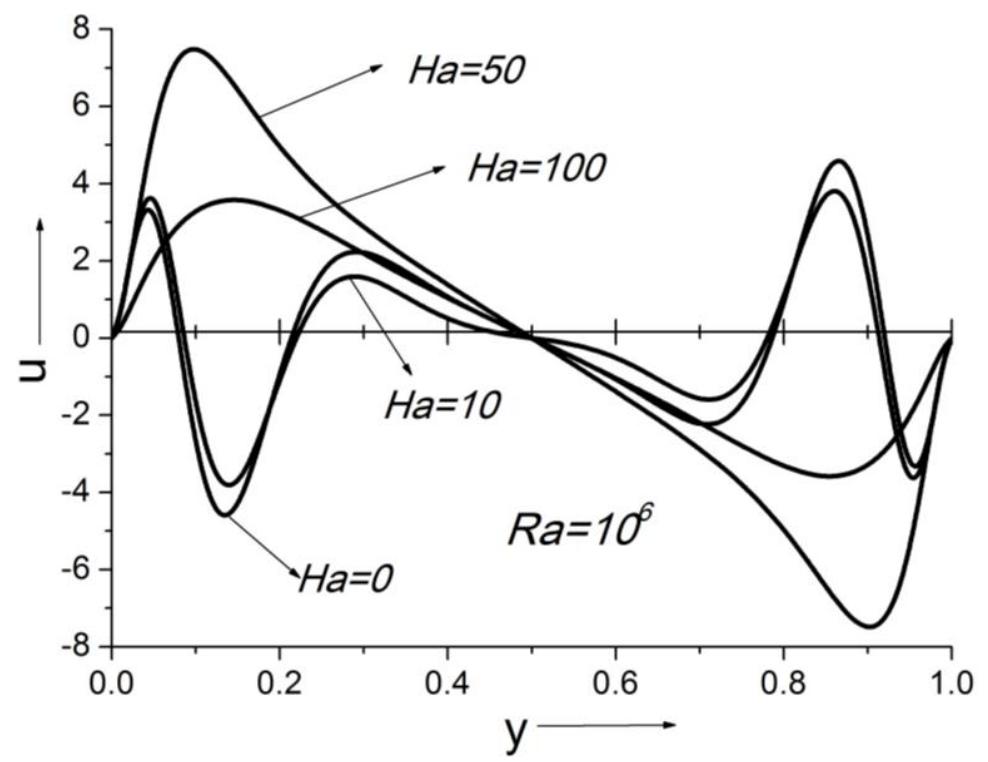

Figure 5. Velocity profiles $u$ at $x=0.5\left(\mathrm{Ra}=10^{6}\right)$.

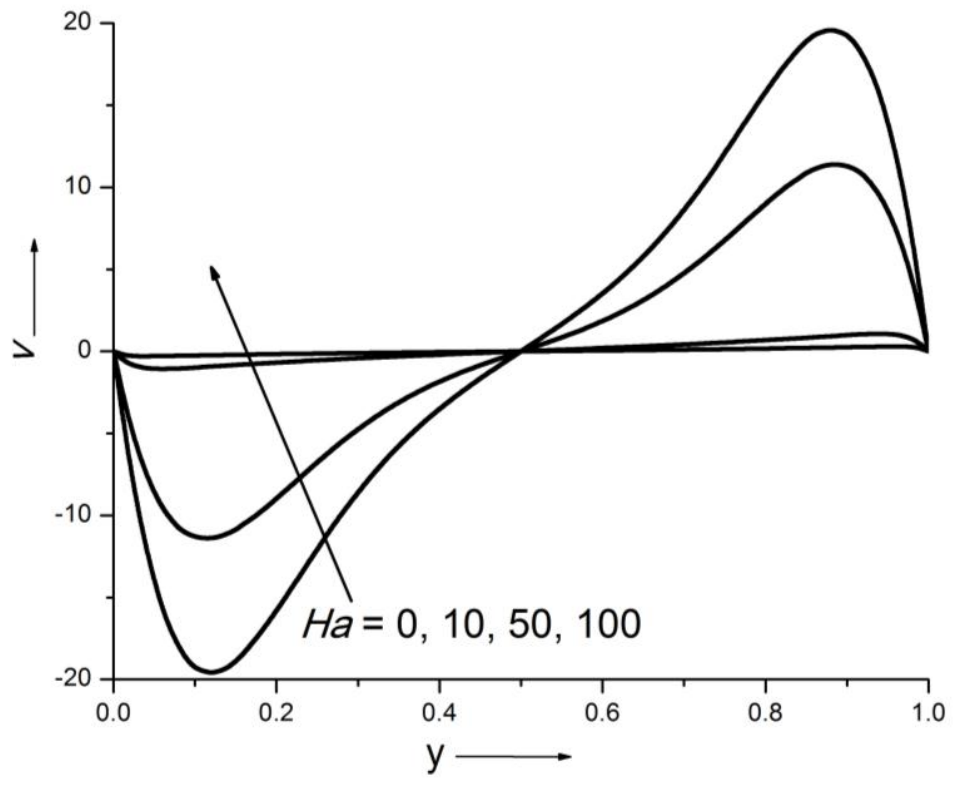

Figure 6. Velocity profiles $v$ at $x=0.5\left(\mathrm{Ra}=10^{4}\right)$. 


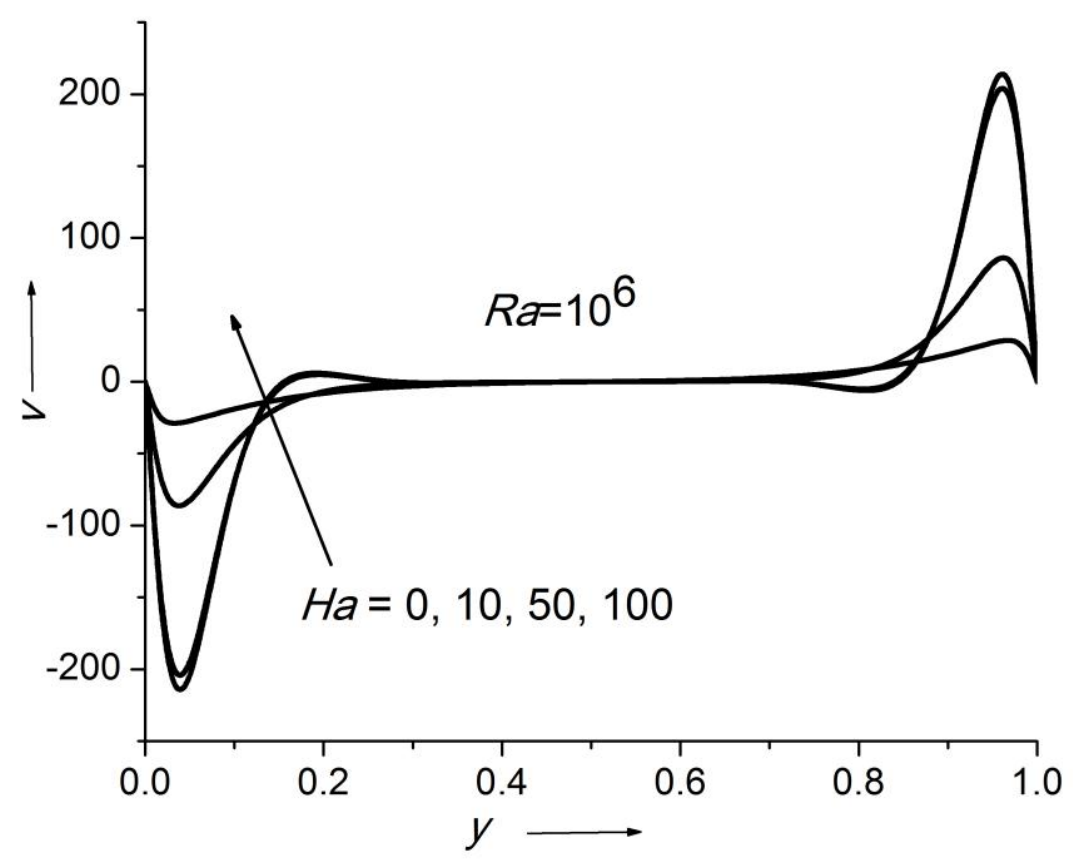

Figure 7. Velocity profiles $v$ at $x=0.5\left(\mathrm{Ra}=10^{6}\right)$.

Since the magnetic field is applied normal to the plane of the cavity, we need to discuss the variation of both components of velocity, $u$ and $v$. In Figures 4 and 5, we show how the $x$-component of velocity, $u$, changes with the vertical coordinate $y$ at the cross-section $x=0.5$, for $\mathrm{Ra}=10^{4}$ and $10^{6}$, respectively. These sketches show that $u$ is oscillatory in nature, with values peaking near the lower and upper adiabatic walls. The seemingly suppressive effect of the applied magnetic field on this component is quite visible for $\mathrm{Ra}=10^{4}$ (Figure 4). However, for larger values of $\mathrm{Ra}$ $\left(10^{6}\right)$, we notice a mixed response of the magnetic field.

In order to assess the combined effect of buoyancy and the magnetic forces on the velocity, we show profiles of the $y$ - component of velocity $v$. The curves are shown at a vertical cross-section $x=0.5$ for $\mathrm{Ra}=10^{4}$ (Figure 6) and $\mathrm{Ra}=10^{6}$ (Figure 7). As observed in a similar study [18], the effect of the increase of magnetic field is to suppress the magnitude of the velocity. As buoyancy force increases from $\mathrm{Ra}=10^{4}$ to $\mathrm{Ra}=10^{6}$, we observe that there is an appreciable variation in the velocity near the adiabatic walls. However, in the central region, the fluid is nearly stagnated for the higher Rayleigh number.

From the profiles of $u$ and $v$, we notice that the magnitude of the vertical component of the velocity is relatively much more influenced by the magnetic field of the type considered here - normal to the plane of the cavity. Furthermore, the flow suppression due to the magnetic field, usually observed in hydromagnetic convective studies, is clearly visible in the velocity component $v$. This, in turn, shows that the convective heat transfer is reduced in the region away from the adiabatic boundaries.

The non-dimensional temperature profiles at the vertical cross-section $x=0.5$ are shown in Figure 8 for the same set of values of $\mathrm{Ra}$ and $\mathrm{Ha}$, as above. Interestingly, at higher values of the Hartmann number $(\mathrm{Ha}=50$ or 100), the temperature varies rather linearly with $y$ when $\mathrm{Ra}=10^{4}$, although showing little variation with the magnetic field. However, for a weak magnetic field, the temperature profiles oscillate about this linear profile, showing opposite behaviour before and after the mid-point $y=0.5$. As the buoyancy force becomes larger $\left(\mathrm{Ra}=10^{6}\right)$, we observe broadly similar features, although in this case the effects of a weak as well as a strong magnetic field are quite clearly manifested. 


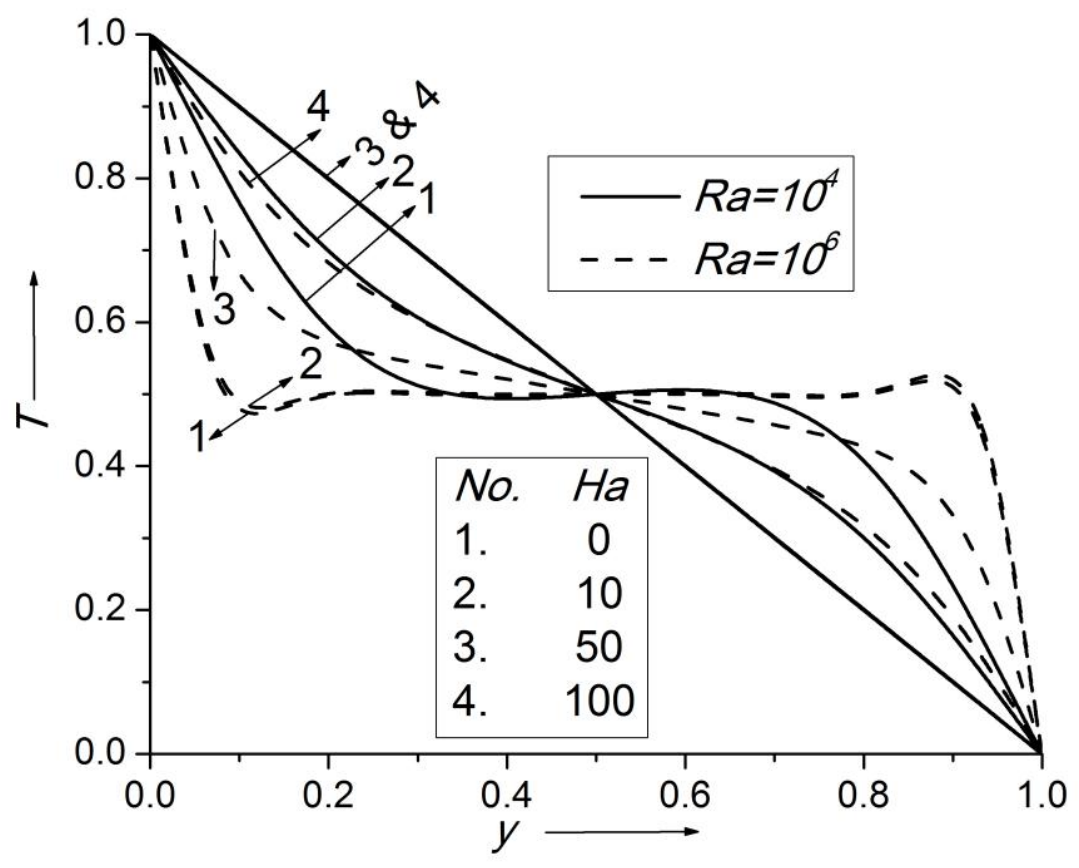

Figure 8. Temperature profiles $T$ at $x=0.5$ for $\mathrm{R} a=10^{4}$ and $\mathrm{R} a=10^{6}$.

In Figure 9, we show the effect of the applied magnetic field on the local Nusselt number $\mathrm{Nu}$. The curves are selfexplanatory. In particular, we note that when the Rayleigh number is low and the Hartmann number high, Nu is nearly constant.

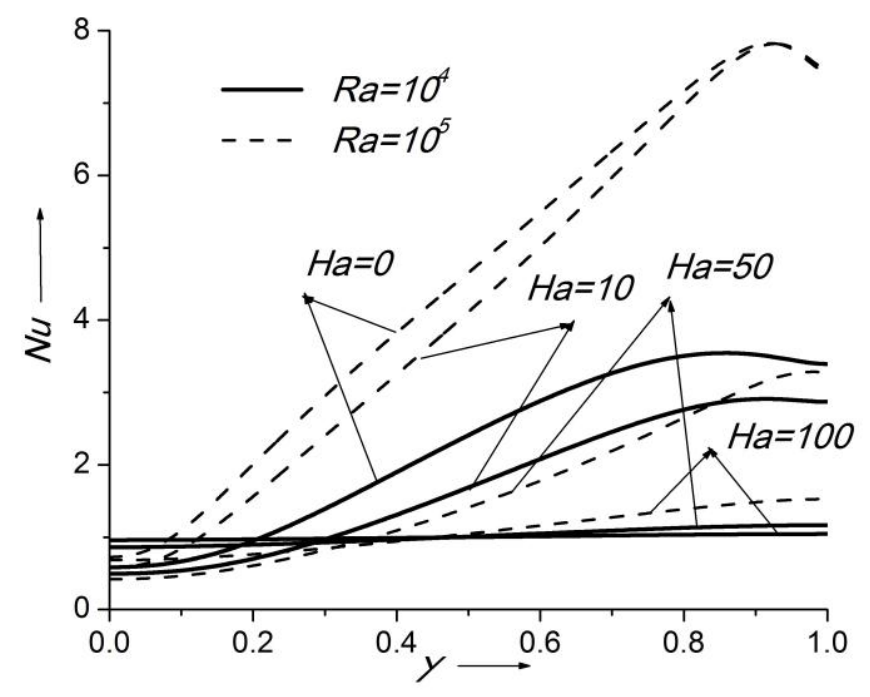

Figure 9. Local Nusselt number at $x=0$ for $\mathrm{R} a=10^{4}$ and $\mathrm{R} a=10^{5}$.

In Table 1, we show the computed values of two parameters of practical importance in applications - the average Nusselt number on the heated wall, $\mathrm{N} u_{\mathrm{av}}$, and the maximum absolute value of the stream function, $|\psi|_{\max }-$ for a range of values of the Rayleigh and the Hartmann numbers. The practical utility of $|\psi|_{\max }$ stems from the fact that it quantifies the intensity of the natural convection in the flow domain. We see from the tabulated values that for a fixed $\mathrm{Ha}$, the effect of increase in $\mathrm{Ra}$ is to increase $\mathrm{N} u_{\mathrm{a} v}$ as well as $|\psi|_{\max }$. On the other hand, when Ra is fixed, $|\psi|_{\max }$ and $\mathrm{N} u_{\mathrm{a} v}$ decrease with Ha. Moreover, for relatively small values of $\mathrm{Ra}, 10^{4} \leq \mathrm{R} a \leq 10^{5}, \mathrm{~N} u_{\mathrm{a} v} \rightarrow 1$ with increasing Ha. 
ANAND KUMAR ET AL

Table 1. Numerical values of $|\psi|_{\max }$ and $\mathrm{N} u_{\mathrm{av}}$.

\begin{tabular}{|c|c|c|c|c|c|}
\hline & & \multicolumn{2}{|c|}{ Present work } & \multicolumn{2}{|c|}{ Previous work [21] } \\
\hline $\mathrm{Ra}$ & $\mathrm{Ha}$ & $|\psi|_{\max }$ & $\mathrm{N} u_{\mathrm{a} v}$ & $|\psi|_{\max }$ & $\mathrm{N} u_{\mathrm{a} v}$ \\
\hline \multirow[t]{4}{*}{$10^{4}$} & 0 & 5.0552 & 2.2879 & 5.05 & 2.29 \\
\hline & 10 & 2.9159 & 1.7091 & 3.35 & 1.97 \\
\hline & 50 & 0.2696 & 1.0105 & 0.47 & 1.06 \\
\hline & 100 & 0.0707 & 1.0008 & 0.12 & 1.02 \\
\hline \multirow[t]{4}{*}{$10^{5}$} & 0 & 9.6884 & 4.5761 & 9.75 & 4.62 \\
\hline & 10 & 8.1514 & 4.1950 & - & - \\
\hline & 50 & 2.2038 & 1.5975 & - & - \\
\hline & 100 & 0.6901 & 1.0710 & 1.14 & 1.37 \\
\hline \multirow[t]{4}{*}{$10^{6}$} & 0 & 16.961 & 8.9214 & 17.0 & 8.90 \\
\hline & 10 & 16.318 & 8.7685 & - & - \\
\hline & 50 & 9.0776 & 5.6404 & 10.51 & 6.39 \\
\hline & 100 & 4.4136 & 2.8784 & - & - \\
\hline
\end{tabular}

This indicates that the heat transfer at high Hartmann number is dominated by conduction which further implies, inter alia, the absence of convection currents in the cavity. On the other hand, for larger values of buoyancy force and weak magnetic field, $|\psi|_{\max }$ attains high values showing the dominance of heat transfer by the convection mode. Finally, in order to assess the effect of the nature of application of the magnetic field - normal to the plane of the cavity vis-à-vis along the plane of the cavity - we have compared our results with that of Pirmohammadi et al. [21]. The broader conclusions drawn above in our analysis are qualitatively very similar to those reported in [21]. As regards the comparative effect due to different modes of application of the magnetic field, we observe that for given Ra and Ha, the values of $|\psi|_{\max }$ and $\mathrm{N} u_{\mathrm{av}}$ are lower in our work as compared to the corresponding values in [21]. This important result of our study shows that the suppression of natural convection in the cavity is enhanced when the magnetic field is applied perpendicular to the cavity in contrast to when it is applied along the cavity in the horizontal direction.

\section{Acknowledgment}

A. K. is thankful to the Council of Scientific and Industrial Research, New Delhi, India for financial assistance in the form of a Senior Research Fellowship.

\section{References}

1. Batchelor, G.K. Heat transfer by free-convection across a closed cavity between vertical boundaries at different temperature. Quart. J. Appl. Math, 1954, 12, 209-233.

2. Eckert, E.R.G. and Carlson, W.O. Natural convection in an air layer enclosed between two vertical plates with different temperatures. Int. J. Heat Mass Transf, 1961, 2, 106-120.

3. Elder, J.W. Laminar free convection in a vertical slot. J. Fluid Mech, 1965, 23, 77-98.

4. Elder, J.W. Numerical experiments with free convection in a vertical slot. J. Fluid Mech, 1966, 24, 823-843.

5. Gill, A.E. The boundary-layer for convection in a rectangular cavity. J. Fluid Mech.1966, 26, 515-536.

6. Wilkes, J.O. and Churchill, S.W. The finite-difference computation of natural convection in a rectangular enclosure. AIChE J, 1966, 12, 161-166.

7. de Vahl Davis, G. Laminar natural convection in an enclosed rectangular cavity. Int. J. Heat Mass Transf, 1968, 11, 1675-1693.

8. Kimura, S. and Bejan, A. The boundary layer natural convection regime in a rectangular cavity with uniform heat flux from the side. J. Heat Transf, 1984, 106, 98-103.

9. Hall, J.D., Bejan, A. and Chaddock, J.B. Transient natural convection in a rectangular enclosure with one heated side wall. Int. J. Heat Fluid Flow, 1988, 9, 396-404.

10. Straughan, B. A nonlinear analysis of convection in a porous vertical slab. Geophys. Astrophys. Fluid Dyn, 1988 , 42, 269-275.

11. Hill, A.A. and Straughan, B. Linear and nonlinear stability thresholds for thermal convection in a box. Math. Meth. Appl. Sci, 2006, 29, 2123-2132.

12. Barletta, A., Magyari, E., Pop, I. and Storesletten, L. Mixed convection with viscous dissipation in a vertical channel filled with a porous medium. Acta Mech, 2007, 194, 123-140. 


\section{EFFECT OF PERPENDICULAR MAGNETIC FIELD ON FREE CONVECTION}

13. Oztop, H.F., Varol, Y. and Pop, I. Effects of wall conduction on natural convection in a porous triangular enclosure. Acta Mech, 2008, 200, 155-165.

14. Tiwari, A.K., Singh, A.K., Chandran, P. and Sacheti, N.C. Natural convection in a cavity with a sloping upper surface filled with an anisotropic porous material. Acta Mech, 2012, 223, 95-108.

15. Straughan, B. Energy Method, Stability and Nonlinear Convection. 2nd Ed. Appl. Math. Sci. Ser., vol. 91 Springer-Verlag, New York (2004)

16. Oreper, G.M. and Szekely, J. The effect of an externally imposed magnetic field on buoyancy driven flow in a rectangular enclosure. J. Cryst. Growth, 1983, 64, 505-515.

17. Garandet, J.P., Alboussiere, T. and Moreau, R. Buoyancy driven convection in a rectangular cavity with a transverse magnetic field. Int. J. Heat Mass Transf., 1992, 35, 741-748.

18. Rudraiah, N., Barron, R.M., Venkatachalappa, M. and Subbaraya, C.K. Effect of a magnetic field on free convection in a rectangular cavity. Int. J. Engng. Sci., 1995, 33, 1075-1084.

19. Alchaar, S., Vasseur, P. and Bilgen, E. Natural-convection heat transfer in a rectangular enclosure with a transverse magnetic field. J. Heat Transf., 1995, 117, 668-673.

20. Kanafer, K. and Chamkha, A. J. Hydromagnetic natural convection from an inclined porous square enclosure with heat generation. Numer. Heat Transf. A., 1998, 33, 891-910.

21. Pirmohammadi, M., Ghassemi, M. and Sheikhzadeh, G.A. Effect of a magnetic field on buoyancy-driven convection in differentially heated square cavity. IEEE Trans. Magnetics, 2009, 45, 407-411.

22. Pirmohammadi, M., Ghassemi, M. and Sheikhzadeh, G.A. Effect of magnetic field on transient natural convection heat transfer. IEEE Trans. Magnetics, 2009, 45, 2788-2790.

23. Al-Najem, N.M., Khanafer, K.M. and El-Refaee, M.M. Numerical study of laminar natural convection in tilted enclosure with transverse magnetic field. Int. J. Numer. Meth. Heat Fluid Flow, 1998, 8, 651-672.

24. Ece, M.C. and Buyuk, E. Natural-convection flow under a magnetic field in an inclined rectangular enclosure heated and cooled on adjacent walls. Fluid Dyn. Res., 2006, 38, 564-590.

25. Pirmohammadi, M. and Ghassemi, M. Effect of a magnetic field on convection heat transfer inside a tilted square enclosure. Int. Comm. Heat Mass Transf, 2009, 36, 776-780.

26. Pirmohammadi, M., Ghassemi, M. and Hamedi, M. Effect of inclination angle on magneto-convection inside a tilted enclosure. IEEE Trans. Magnetics, 2010, 46, 3697-3700.

27. Pirmohammadi, M. and Ghassemi, M. Numerical study of magneto-convection in a partitioned enclosure. IEEE Trans. Magnetics, 2009, 45, 2671-2674.

28. Pirmohammadi, M., Ghassemi, M. and Keshtkar. A. Numerical study of hydromagnetic convection of an electrically conductive fluid with variable properties inside an enclosure. IEEE Trans. Plasma Sci., 2011, 39, 516520.

29. Mallinson, G.D. and de Vahl Davis, G. The method of the false transient for the solution of coupled differential equations. J. Comp. Phys., 1973, 12, 435-461.

30. Samarskii, A.A. and Andreyev, V.B. On a high-accuracy difference scheme for an elliptic equation with several space variables. USSR J. Comp. Math. Math. Phys., 1963, 3, 1373-1382.

Received 18 April 2014

Accepted 22 January 2015 